ESTELLA FREIRE

CARMEN FRANCO-VÁZQUEZ

CANDELA RAJAL ALONSO
Universidad de Santiago de Compostela

estela.freire@usc.es

carmen.franco@usc.es

candelarajal@gmail.com

\title{
Contramapas del Camino. Memorias del habitar
}


COUNTER-MAPS OF THE “CAMINO”. MEMORIES OF THE WAY OF LIVING.

\section{ABSTRACT}

The following article highlights the importance of educating in the context and in common life for the construction of a collective memory, reestablishing identity bonds, preserving the past of our communities and reformulating the present. Through the educational project Contramapas del Camino. Memories of the way of living, carried out in different rural contexts belonging to the Camino de Santiago, we propose a series of artistic actions and methodologies that involve the inhabitants in the processes of recovery of memory linked to these places. This project, developed by the Didactics of Visual Expression Department of the University of Santiago de Compostela, is part of the $O$ teu Xacobeo programme promoted and financed by the Xunta de Galicia. The main objective is to empower and make more visible the role of Artistic Education and creative processes as tools of analysis in the development of interdisciplinary proposals. The panels presented as Cartografías de la Memoria Popular (Cartographies of Popular Memory), synthesize the graphic-visual documentation generated from the different workshops and from the productions and narratives analyzed.

\section{Keywords}

Collective memory; Identity; Heritage; Art Education; Environmental Education.

\section{RESUMEN}

El siguiente artículo pone de manifiesto la importancia de educar en el contexto y en la vida en común para la construcción de una memoria colectiva, restableciendo los vínculos identitarios, preservando el pasado de nuestras comunidades y reformulando el presente. A través del proyecto educativo Contramapas del Camino. Memorias del habitar llevado a cabo en diferentes contextos rurales pertenecientes al Camino de Santiago, se proponen una serie de acciones y metodologías artísticas que implican a los habitantes en los procesos de recuperación de la memoria vinculada a estos lugares. Este proyecto, desarrollado desde el Departamento de Didáctica de la Expresión Plástica de la Universidad de Santiago de Compostela, forma parte del programa $O$ teu Xacobeo impulsado y financiado por la Xunta de Galicia. El objetivo principal es el de potenciar y hacer más visible el papel de la Educación Artística y de los procesos creativos como herramientas de análisis en el desarrollo de propuestas interdisciplinares. Los paneles presentados como Cartografías de la Memoria Popular sintetizan la documentación gráfico-visual generada a partir de los diferentes talleres y de las producciones y narrativas analizadas.

\section{Palabras clave}

Memoria colectiva; Identidad; Patrimonio; Educación Artística; Educación Ambiental. 
El contexto rural gallego es un contexto de fragmentación donde entra en conflicto lo geográfico, lo económico y lo social. La población gallega se caracteriza por habitar en núcleos pequeños; son pueblos y villas con reducido número de habitantes, ya que la mayoría de la población vive dispersa en aldeas o pequeños agrupamientos de casas. Sánchez Pardo (2013), afirma que "la estructura de poblamiento rural en Galicia no se ajusta a criterios y ordenaciones válidas en otras zonas de España" (p. 77). Hablamos de territorios muy afectados por el envejecimiento poblacional y el abandono a lo que se une el carácter y la dispersión geográfica del rural gallego anteriormente descrita. Galicia se caracteriza por ser una de las comunidades autónomas de España, junto con Castilla y León y Asturias, con la mayor proporción de personas mayores de 64 años (INE, 2012). La esperanza de vida de su población al nacer era de 84,03 en el año 2018 (IGE, 2020); estamos ante lo que Aldrey y do Rio (2014) denominan "envejecimiento sumamente acusado".

Estas problemáticas hacen que el territorio y su herencia identitaria se vean desprotegidos, centrando los puntos de interés y conservación patrimonial en áreas más pobladas y urbanas. En el caso del Camino de Santiago nos encontramos con pequeñas poblaciones vinculadas a las Rutas Xacobeas donde se tiende a la invisibilización de la memoria colectiva en pro de la conservación y protección de bienes relacionados con el Camino y de interés turístico, estableciendo un relato uniformizado e institucionalizado que deja atrás los relatos de la memoria de los propios habitantes. Al perderse ese vínculo con el contexto está en peligro no sólo la preservación del pasado sino también los vínculos identitarios que dan sentido a las comunidades en el presente.

Surge de esta forma Contramapas del Camino. Memorias del habitar, un proyecto de intervención e investigación educativa para la recuperación de la memoria colectiva en cuatro ayuntamientos de la comarca de Santiago de Compostela: Santiago, Teo, Ames y Touro. Una propuesta de carácter horizontal e intergeneracional que plantea, teniendo como pilares la Educación Artística y la Educación Basada en el Lugar, la generación de experiencias que rescaten el patrimonio colectivo a través del intercambio, la reflexión y la acción. Una forma de comprender el patrimonio de una forma viva y flexible para resignificar nuestras realidades presentes y futuras.

En este artículo ponemos de manifiesto la necesidad de restablecer vínculos con nuestro entorno para la reconstrucción de nuestra memoria colectiva y para tomar acción sobre las propias necesidades que demandan los lugares que habitamos en nuestro día a día. Centraremos nuestro punto de interés en las metodologías empleadas en el proyecto, extrapolables a otros contextos, que ponen a las personas que habitan en los mismos en primer plano, dándoles voz y visibilizando sus historias de vida, sus experiencias, sus emociones y a sus intereses. 


\section{MARCO TEÓRICO}

2.1 Construcción de la memoria colectiva a través del patrimonio de lo cotidiano

Para la construcción de una memoria colectiva es necesario valorar y comprender aquello que vivimos y compartimos como cotidiano. Las imágenes mentales y experiencias que nos son comunes y que configuran nuestros recuerdos y nuestra historia, constituyen un patrimonio personal que se comparte como patrimonio colectivo.

Todo bien patrimonial formó parte de una experiencia cotidiana común en un espacio y tiempo determinado. El patrimonio está constituido desde una dimensión social (Fontal, 2004) y somos las personas las que le otorgamos importancia y trascendencia. Muñoz-Viñas (2010) destaca que existen un tipo de bienes que tienen un carácter simbólico porque sobre ellos "se vuelcan unos valores que en realidad corresponden a sentimientos, creencias o ideologías, es decir, a aspectos inmateriales de la realidad" (p.40). Estos bienes no tienen por qué ser materiales y su significado es acordado y concedido por un grupo de personas, o incluso, en ciertos casos, por una sola persona (p.40).

Este patrimonio es intangible pero realmente está en todos los aspectos de los bienes culturales y es la base de la identidad, la creatividad y la diversidad cultural (Arévalo, 2009). Todas las cosas que hemos ido atesorando
como individuos, ya sea un recuerdo, una canción, una danza, una lengua, un proverbio, una imagen, un lugar, una persona o un objeto; todo cuanto nos rodea es susceptible de ser patrimonio, o en su actual efecto, patrimoniable (Fontal, García-Ceballos, Aso Morán y Martínez, 2017, p.5).

Podríamos hablar de que la suma de estas expresiones nos define como personas. El patrimonio de la vida es conformador de nuestra identidad y constructo como individuos (Fontal et al., 2017), un patrimonio de lo cotidiano, que pone de manifiesto la importancia de objetos, relaciones, actividades y acontecimientos a los que les otorgamos cierta importancia y que son fruto de nuestra experiencia.

Según Halbwachs (2004) para la constitución de la memoria colectiva es clave el milieu de mémoire o entorno, el espacio compartido por la comunidad y todo lo que la rodea. La interdependencia entre la memoria individual y la colectiva, son claves que nos permiten

hablar de memoria colectiva cuando evocamos un hecho que ocupaba un lugar en la vida de nuestro grupo y que hemos planteado o planteamos ahora en el momento en que lo recordamos desde el punto de vista de este grupo (p.36).

Es primordial trabajar en el presente por la revalorización de las expresiones de identidad comunitaria. A partir de la conservación y transmisión de la memoria colectiva, podremos llevar a cabo una apropiación social del patrimonio y tomar conciencia de su trascendencia en nuestras realidades. 
experiencias, vivencias y, sobre todo, otras personas" (Fontal y Marín-Cepeda, 2018, p.485).

Nuestra experiencia cotidiana comunitaria y los lugares que forman parte de nuestro día a día son detonantes de nuestra memoria colectiva, ya que es en el espacio donde nos imaginamos la existencia sensible de aquellos con los que nos relacionamos, de viva voz o por gestos en un momento dado (Halbwachs, 2004). Así pues, podemos decir que es en los lugares donde habita la memoria, porque

no hay memoria colectiva que no se desarrolle dentro de un marco espacial. Ahora bien, el espacio es una realidad que dura: nuestras impresiones se expulsan una a otra, nada permanece en nuestra mente, y no comprenderíamos que pudiéramos recuperar el pasado si no lo conservase el medio social que nos rodea (Halbwachs, 2004, p.147).

Para esta preservación es necesaria una revisión continua del pasado en el presente por los habitantes de la propia comunidad. Esta relectura hace del patrimonio algo vivo que se hereda y se transmite, experimentando cambios, creándose y recreándose, siendo una de sus características la capacidad de transformación y la creación de vínculos generacionales (Arévalo, 2010). Nuestros relatos cobran importancia al solaparse los unos con los otros en el pasado y en el presente.

Según Assmann (2010) existe la memoria del recuerdo vivo que se configura en la comunicación cotidiana a través del lenguaje, desde el intercambio y la experiencia común, de forma espontánea sin necesidad de soportes institucionales uniformizadores, en un proceso continuo inacabado de transmisión. Tal y como cita Arévalo (2009):

El patrimonio intangible está en todos los aspectos de los bienes culturales. Y es la base de la identidad, la creatividad y la diversidad cultural. Es un patrimonio vivo, continuamente recreándose, que cobra vida a través de los seres humanos y de sus prácticas y formas de expresión (p. 172).

Las acciones y experiencias interpersonales e intergeneracionales en el presente son claves para este proceso de transmisión, para valorar y preservar el pasado, y para resignificar el presente. Esto forma parte de lo que Fontal y Marín-Cepeda (2018) denominan como un andamiaje identitario que está en continuo proceso de construcción a través de vínculos de identidad, propiedad, pertenencia y emoción, que son necesarios para formar la personalidad y construir la interacción social de los individuos. Es precisamente desde las relaciones socioafectivas desde donde las personas damos sentido a nuestro entorno.

\footnotetext{
Las personas, grupos y comunidades son actores activos que se encuentran en un continuum sobre la construcción de la historia. Así mismo, las personas son las contenedoras de las experiencias, las narrativas de vida, a modo de tesoros vivos de la cultura, de testigos de la misma, poseen conocimientos y experiencias que deben ser preservados, para su conocimiento y transferencia futura (Fontal et al., 2017, p.8).
}

Por una parte, es necesario incorporar los relatos invisibilizados por la Historia a la memoria colectiva y que las nuevas generaciones comprendan este legado y su renovación porque sólo así se asentará nuestro relato colectivo y nuestra identidad. Por otra parte, debemos trabajar también el relato actual porque forma parte de nuestro patrimonio presente, y debemos 
cuidar el legado que estamos construyendo para sumarlo a ese hilo de la transmisión y dejarlo en herencia a generaciones que necesitarán saber qué se hizo hoy, por qué y para qué, dado que ellas serán pura herencia de nosotros (Fontal, 2013, p.75).

Este fuerte componente generacional en la transmisión de la memoria identitaria unido a una educación en sociedad pueden ser decisivos para esta lucha de reapropiación del patrimonio cultural.

Este patrimonio cultural inmaterial, que se transmite de generación en generación, es recreado constantemente por las comunidades y grupos en función de su entorno, su interacción con la naturaleza y su historia, infundiéndoles un sentimiento de identidad y continuidad y contribuyendo así a promover el respeto de la diversidad cultural y la creatividad humana (UNESCO, 2003).

Desde el ámbito educativo se pueden generar experiencias significativas que partan del propio contexto y de los propios agentes que forman parte de él, para repensar el pasado y generar nuevas herramientas para transformar nuestra realidad en el presente y en el futuro.

\subsection{Lugar e Identidad: la educación basada en el lugar a través de metodologías artísticas}

Este proyecto parte del estudio del lugar y de su herencia cultural mediante el diseño de propuestas educativas que toman como base los principios de los movimientos AEE (Arts-based Environmental Education) y $A B E$ (Art and the Built Environmental). Estas propuestas exploran la relación entre la educación artística y la educación ambiental a través de la experiencia directa con el contexto. Los objetivos que persiguen este tipo de metodologías son, principalmente, mejorar la respuesta emocional al lugar y desarrollar habilidades críticas y de diseño que permitan afrontar cambios en nuestro entorno inmediato (Adams, 1989).

Hablar de estrategias educativas ligadas al estudio del contexto permite desarrollar procesosproyectos de aprendizaje que parten de las necesidades culturales, medioambientales y políticas derivadas del análisis del espacio natural y construido que nos rodea. Educar a las personas en relación a su contexto inmediato permite indagar sobre nuevas formas de "acercamiento a la experiencia y comprensión de las cualidades del entorno" (Palacios, 2006, p.65).

Para articular y estructurar toda la información subyacente al análisis de un lugar, podemos recurrir a diversas técnicas. La actividad creativa es una herramienta clave para interconectar la experiencia vivencial con la significación que obtenemos de dicha experiencia. En un mundo dominado por la tecnología digital, debemos priorizar el valor educativo de la experiencia. Dewey en El Arte como Experiencia (2008) expone que la naturaleza de la experiencia está determinada por las condiciones esenciales de la vida diaria, y esta vida se produce no sólo en un ambiente determinado, sino a causa de éste y a través de la interacción de la experiencia con el entorno. En esta línea, Meri-Helga Mantere (1992) apunta las siguientes reflexiones sobre las relaciones entre experiencias educativas y entorno: 
El sistema educativo debe garantizar esta conexión con el entorno a través de una educación basada en la experiencia y en la reflexión sobre la acción de dicha experiencia. El concepto de experiencia definido por Dewey (2004) hace referencia a la interacción constante entre el individuo y su ambiente, a la interconexión entre los hechos del pasado y del presente. Según el autor "el problema central de una educación basada en la experiencia es seleccionar aquel género de experiencias presentes que vivan fructífera y creadoramente en las experiencias subsiguientes" (p.73).

El arte se presenta pues como una estrategia facilitadora del entendimiento de la experiencia dando forma a nuestras ideas con el fin de cristalizar, simbolizar y comunicar pensamientos, valores y sentimientos (Adams, 2012). Necesitamos alimentar la sensibilidad estética desde el ámbito social y educativo, garantizando la transmisibilidad del respeto hacia nuestro entorno a través del reconocimiento consciente y sensible de los factores que determinan sus transformaciones. Adams (1998) al igual que Dewey, sitúa como primordiales aquellos aspectos ordinarios y cotidianos de nuestro entorno que en principio suelen pasar desapercibidos.

Debemos acercarnos a un reconocimiento consciente de los factores que determinan las transformaciones de nuestro entorno para poder valorarlo, respetarlo, cuestionarlo y transformarlo. En este sentido, la experiencia estética y la práctica artística sobre el entorno constituyen, como citan Blanco y Cidrás (2019), un proceso de exploración de las calidades del propio entorno. La creación artística se presenta como una herramienta clave que "nos invita a hacernos preguntas, a buscar alternativas y a generar nuevas relaciones y actuaciones con la realidad" (p.35). El análisis plástico y visual del entorno y de sus componentes permite analizar las casuísticas particulares de los lugares que habitamos y en los que desarrollamos la actividad cotidiana del habitar.

El registro plástico y visual ligado al registro verbal y escrito (cuadernos de viaje, narrativas, fotografías, dibujos de campo) son recursos que posibilitan un entendimiento natural de la información obtenida a través de diferentes niveles perceptivos. A través de prácticas artísticas y mapeos colectivos sobre el territorio, la comunicación plástica y visual se convierte en un lenguaje común que permite la exploración de nuevas formas de unión entre las personas y el contexto en el que viven (Illeris, 2012). Estas formas de educación basada en el lugar (Gruenewald \& Smith, 2008) permiten aumentar la comprensión y el uso de las dimensiones del lugar como herramientas educativas para la sostenibilidad y el respeto por el entorno.

Una exploración consciente de nuestro entorno posibilita el reconocimiento de las múltiples formas de ocupación y de los modos de habitar que se dan en el territorio. Descubrir nuestro entorno de forma local permite un acercamiento a lo global desde el reconocimiento de una identidad propia que es inherente al lugar en el que esa identidad se desarrolla. La intención es nutrir la conciencia y la sensibilidad para reflexionar sobre las formas de gestionar y modificar nuestro entorno mediante metodologías que vayan más allá de los aspectos formales, e integren los valores y cuestiones culturales propias de dicho entorno (Adams, 1998).

Existe una necesidad de que los estudios sobre el entorno tengan en cuenta los contenidos sociales, culturales e interculturales para poder redefinir el acercamiento al entorno y reconsiderar críticamente los modelos que rigen nuestra relación con el ambiente (Palacios, 2005). Palacios insiste en la necesidad de enfocar de forma interdisciplinar el estudio del entorno natural y construido a través de las diferentes perspectivas que ofrecen campos como el arte, la educación, la arquitectura, la ecología, la sociología y el activismo social. La indagación 
sobre este tipo de educación permite explorar estrategias educativas ligadas directamente a las comunidades, focalizando los recursos de las escuelas, centros sociales y universidades como catalizadores para el tratamiento de problemas reales en el entorno inmediato. La educación basada en el contexto difiere de la educación tradicional hermética en el sentido en que se formula desde las experiencias personales y locales de los estudiantes en relación a las problemáticas que experimentan en su entorno (Jokela y Coutts, 2018).

Se trata de presentar diferentes enfoques pedagógicos que articulen la experiencia educativa con una voluntad de transformación sostenible de la realidad. Helene Illeris (2012) acuñó el concepto Educación Artística para el Desarrollo Sostenible (AESD) presentándola como un proceso de mapeo que la autora establece como primordial para comprender cómo las recientes corrientes en la educación artística pueden contribuir al desarrollo de la sostenibilidad ambiental. El desarrollo sostenible del entorno en que vivimos no se refiere solamente a su conservación, sino que, tal y como señala Mantere (1992), el cambio se genera a través de la creación de nuevas posibilidades de relación en el entorno.

Estas perspectivas pedagógicas enlazan con la línea principal de investigación que motivó el diseño y desarrollo del proyecto que se presenta. Entendemos el proceso de creación artística sobre el entorno como instrumento clave para la reflexión sobre el propio entorno. La utilización de diferentes métodos artísticos para la comunicación de las experiencias relativas al estudio, análisis y transformación del entorno, favorece el desarrollo de una actitud crítica, reflexiva y activa en relación a él (Neperud, 1978). En este sentido, todas las prácticas presentadas convergen en una única dirección que es la de concienciar a la población sobre su papel activo y en las alternativas de transformación sostenible que los diferentes colectivos aportan cuando se trabaja en comunidad. Partir del estudio del local en todas sus dimensiones y diseñar propuestas de aprendizaje según las necesidades particulares de los que habitan, es apostar por una educación centrada en el contexto que articule el pensamiento divergente, la actitud creativa y las posibilidades de acción para la mejora sostenible de nuestro entorno.

\section{CONTRAMAPAS DEL CAMINO: MEMORIAS DEL HABITAR}

\subsection{Presentación y objetivos}

Contramapas del Camino. Memorias del habitar, es una propuesta de acción para la recuperación y puesta en valor del patrimonio natural, material e inmaterial ligado a los territorios rurales del entorno de Santiago de Compostela. De este tejido emergen estructuras de fuerte componente identitario que se mantienen y se consolidan como elementos patrimoniales de especial valor material e inmaterial ligados a los ejes por los que discurren los diferentes Caminos que conforman la Red Xacobea. Los caminos que conforman la Red Xacobea son, por definición, lugares de intercambio y sociabilización. Su evolución, transformación y visibilización está en relación directa con la identidad de los habitantes, y son por lo tanto excelentes transmisores de la historia del lugar y de su memoria. El proyecto se desarrolla en cinco áreas rurales diferenciadas pertenecientes a la comarca de Santiago de Compostela. Las zonas de actuación incluyen además del ayuntamiento de Santiago de Compostela los ayuntamientos de Teo, Ames y Touro.

Se trata de hacer una reflexión sobre el estado estético-social del camino desarrollando talleres que liguen las comunidades de vecinos a través de la educación artística y patrimonial, 
inventariando la documentación resultante. Este tipo de propuestas educativas se traducen en talleres de formación y concienciación basados en el intercambio de experiencias y en la búsqueda de oportunidades para la divulgación, la reflexión y el pensamiento crítico y cívico de cara a los espacios de los que formamos parte.

El proyecto parte de la necesidad de creación de redes activas entre las comunidades de habitantes para la puesta en valor del patrimonio cultural, natural y artístico que define estos lugares. Las metodologías artísticas empleadas en los diferentes talleres se presentan como principal herramienta para el análisis y reflexión. De esta forma el proyecto persigue como objetivo principal evidenciar el papel fundamental de los procesos artísticos en el desarrollo de propuestas interdisciplinares. El análisis de las diferentes producciones artísticas generadas en los talleres realizados (narrativas, objetos, maquetas, fotografías...) permite elaborar una documentación final en un panel de gran formato que representa esa visión particular que sobre el territorio tienen las personas que habitan en los bordes de los Caminos.

El proyecto se centra en los siguientes objetivos específicos:

1. Promover y divulgar el patrimonio natural y cultural en torno al camino. Contramapas del Camino trabaja el relato oral de los habitantes de la zona, donde se entremezclan narrativa y paisaje, donde las voces de los habitantes permiten construir el imaginario colectivo asociado al territorio. La recogida de datos sobre el patrimonio natural y construido a través de las historias de vida de sus habitantes, permite identificar elementos culturales olvidados poniendo en valor oficios, músicas, construcciones, tradiciones y modos de hacer que constituyen la identidad y refuerzan el sentido de pertenencia.

2. Crear redes de intercambio social y cultural, destacando el patrimonio humano y el intercambio de experiencias. Esta propuesta parte de la necesidad de comprender los espacios del Camino desde su dimensión social y cultural. A través del proyecto educativo presentado y del plan de divulgación llevado a cabo por los grupos de investigación que participan, se pretende dar a conocer nuevos aspectos relacionados con el patrimonio y la construcción de la identidad y cultura colectivas.

3. Poner en valor el arte y la educación artística como herramienta de sensibilización y acercamiento a nuestro entorno. Las estrategias y procesos artísticos nos permiten trabajar desde la experiencia individual y colectiva, y desde un enfoque crítico y reflexivo sobre el territorio. Trabajar con una metodología ligada a las artes permite un mayor acercamiento al patrimonio desde la propia experiencia y desde nuestra experiencia como grupo.

4. Impulsar el desarrollo y la puesta en valor de áreas rurales con una tradición vinculada directamente a los Caminos, pero que no forman parte de los núcleos turísticos principales. El proyecto se desarrolla en zonas de carácter rural visibilizando las experiencias de intercambio y convivencia entre la población y los peregrinos que han sido significativas para la comunidad y para la formación del territorio. 


\subsection{Metodología}

Se trata de un proceso de investigación-producción colectiva donde los procesos de autoformación y retroalimentación dan pie a procesos más complejos de investigación, creación y coaprendizaje. Esta investigación, de corte exclusivamente cualitativo, trabaja desde una perspectiva construccionista que se desarrolla a partir del análisis de las relaciones entre habitantes y contexto a través de la práctica artística. La investigación parte del estudio del contexto inmediato y lo relaciona con el compromiso y la necesidad de generar un conocimiento basado en la experiencia cotidiana de las personas (Taylor y Bogdan, 1996).

Emplearemos por ello la Investigación Basada en las Artes (IBA), ya que nos permite analizar la experiencia a partir de las propias producciones artísticas de los sujetos implicados en el estudio (McNiff, 2008). Las manifestaciones artísticas visibilizan diferentes perspectivas de un mismo acontecimiento abordándolo desde su complejidad. No se trata de llegar a una certeza sino de buscar diversas formas de mirar y representar la experiencia (Barone y Eisner, 2006). Tratándose de recuperar la memoria colectiva, el uso del arte como herramienta y metodología nos permite tener en cuenta cada narrativa y nos aporta otro tipo de datos personales y vivenciales que no aportan otro tipo de fuentes escritas o numéricas (Barone y Eisner, 2006).

Este tipo de investigación analiza las producciones y narrativas aplicando múltiples métodos de evidencia y recolección de datos. El reconocimiento de la experiencia artística como metodología de investigación permite teorizar la práctica de las artes visuales situándola en relación a tres paradigmas: el interpretativo, el empirista y el crítico (Sullivan, 2004). En general, se considera que lo que caracteriza el componente estético de este tipo de investigación no es una cualidad inherente y esencial, sino que parte de un contexto social más amplio (Barone, 2003). Mullen (2003) señala que uno de los propósitos de la IBA es centrarse en el proceso de cómo expresar el contexto de las situaciones vividas más que en mostrar los productos finales desconectados del contexto de su creación, en torno al cual siempre surgen diferencias en la forma de interpretar y analizar sus significados. Considerar, como indica Marín (2011), la educación artística como un territorio de investigación con su propia identidad distintiva, permite ubicar la investigación justo en la intersección entre los problemas de las artes visuales y los problemas de la educación.

Este tipo de investigaciones emplean generalmente un enfoque multimetódico (Denzin y Lincoln, 1994) para el análisis y la recopilación de datos. Según Yin (2003) la teoría resultante puede convertirse en el vehículo para examinar otros casos y nuevas investigaciones empíricas en otros lugares. Se trata de analizar cada caso desde su contexto real, donde los límites entre el fenómeno y su contexto son difusos. No se trata de hacer generalizaciones sino de estudiar cada caso holísticamente buscando aspectos comunes que beneficien el desarrollo de proposiciones teóricas aplicables a otros contextos.

La necesidad de buscar otras formas de recuperación y puesta en valor del patrimonio cotidiano mediante intervenciones y acciones artísticas exige resituar la importancia de la educación y de la transmisión del conocimiento y de la cultura. Este cambio conviene hacerlo teniendo en cuenta los movimientos sociales, la producción cultural y las acciones de colaboración y cooperación. En este sentido, la propuesta se basa en el diseño de prácticas colaborativas donde diferentes grupos interactúan favoreciendo el intercambio y la transmisión de conocimiento intergeneracional. 
El punto de partida es la reunión en los diferentes centros sociales con el colectivo vecinal con el objetivo de obtener información de primera mano sobre la problemática del lugar, sus condicionantes y sus formas de vida. Estos grupos están formados por personas mayores, en su mayoría jubiladas, que acuden a las actividades culturales de los centros, que pueden aportar una experiencia del lugar basada en un enfoque histórico. Esta información será trasladada a las nuevas generaciones, alumnado de Educación Primaria e Infantil, mediante talleres realizados en colaboración directa con los centros educativos más cercanos. El intercambio generacional es clave para comprender la memoria de los lugares a través de la reinterpretación de su pasado y su presente.

Los talleres se diseñan según los principios expuestos en el marco teórico, combinando la educación artística aplicada al contexto y la educación ambiental y sostenible del territorio. Parece una necesidad urgente recuperar la conexión directa de la comunidad educativa con el contexto local, haciendo partícipes a las nuevas generaciones de la construcción de la identidad colectiva del lugar en el que vive.

\subsection{Diseño y desarrollo}

Las acciones propuestas son acciones pensadas para conectar al individuo en sociedad, esto es, en relación directa con el entorno natural y construido y con el resto de comunidades que las habitan. Las reuniones con el vecindario permiten la recogida de documentación sobre la memoria del lugar para la elaboración posterior de los talleres en los que se trabajará la sensibilización y el potencial estético y social de los lugares del Camino. Las experiencias y producciones derivadas de los talleres y de los paseos organizados por los lugares analizados e inventariados se traducen en una cartografía social de los que habitan, en una carta de presentación para los que transitan.

Se ofreció el proyecto a cuatro ayuntamientos del área metropolitana de Santiago de Compostela, abarcando los territorios próximos a los caminos más relevantes de la red Xacobea (Camino Francés, Camino Inglés, Camino Holandés, Camino Portugués y el Camino a Fisterra). Las actividades propuestas fueron incluidas dentro de la programación sociocultural de cada municipio, gestionada en colaboración con los diferentes colectivos y asociaciones vecinales. Dichas asociaciones cívicas tienen diferente denominación según se trate de Ames (locales sociales), Santiago (centros socioculturales), Teo (casa común) o Touro (casa de cultura).

La secuencia de actividades queda reflejada en los puntos siguientes:

Actividad 1. Reunión en el centro social con el colectivo vecinal para recopilar información sobre lo que es, fue y será la vida ligada a los diferentes lugares de los Caminos de la Ruta Xacobea.

Actividad 2. Construcción de un mapa mental-emocional de los espacios en los que vivimos a través de objetos y fotografía personales que liguen memoria, identidad y lugar.

Actividad 3. Presentación en los centros educativos mediante rutas guiadas a los lugares más significativos del contexto. Esta presentación se realizará en colaboración con los centros sociales y los asistentes a las jornadas previas de recogida de información. 
Actividad 4. Desarrollo de la propuesta de trabajo en el centro educativo ligada al campo de la educación artística en base al análisis del entorno trabajado. Se llevarán a cabo varios talleres de experimentación y reflexión sobre el contexto con el objetivo de presentar diferentes perspectivas de análisis y propuestas de alternativas.

Actividad 5. Elaboración de una Cartografía de la Memoria Popular, un Contramapa impreso en gran formato que recoja y sintetice las diferentes experiencias y producciones con el objetivo de presentar y divulgar la información entre el resto de habitantes y transeúntes del lugar.

Con este mapa de ruta se pretende abordar el análisis de las problemáticas específicas que afectan a cada territorio y su impacto en una muestra intergeneracional que ofrezca una visión global de la relación entre los habitantes y el entorno. Los datos derivados de las narrativas y las producciones (collages, dibujos, maquetas y fotografías), permiten la comparación entre las diferentes experiencias analizadas, lo que nos hace reflexionar sobre las posibilidades de transferibilidad del estudio en contextos similares. En referencia a la elección de la muestra, Samaja (1994) define este concepto como cualquier subconjunto de un universo bien definido. Una buena muestra, tal y como cita Samaja, no responde a criterios absolutos, está en relación directa con la estrategia investigativa y con el contexto. Las unidades de análisis son las cuatro primeras actividades definidas en la secuencia de acciones que se desarrollaron en cada lugar y centro especificados a continuación. La actividad 5 se considera como propuesta de síntesis de las actividades anteriores, elaborada por el propio grupo de investigación.

\subsection{Unidad de análisis 1. Lavacolla. La Fábrica de Curtidos y su historia. Camino Francés}

- Centros de realización: Centro Sociocultural de Lavacolla y CEIP Rodríguez Xixirei.

- Número de participantes: 10 mujeres del grupo de Memoria Activa del Centro Sociocultural/ Una clase de 50 de Primaria (18 personas).

- Duración de la propuesta: Sesión de 1 h en el centro sociocultural para las actividades 1 y 2 / 2 sesiones de 50 min para las actividades 3 y 4 desarrolladas en el colegio*.

- Eje principal de desarrollo de la propuesta: Antigua Fábrica de Curtidos.

*Visita guiada por las vecinas a la Antigua Fábrica y desarrollo de una maqueta y cartografía sobre nuevas alternativas de uso de este espacio y su relación con el entorno.

- Desarrollo de la propuesta: En el caso del núcleo de Lavacolla, la propuesta se centra principalmente en el estudio y análisis de la antigua Fábrica de Curtidos. Este complejo, compuesto por varias edificaciones, recoge entre sus paredes la herencia de la memoria histórica del núcleo. Trabajar la evolución de los espacios interiores y plantear nuevos usos mediante una maqueta a escala $1 / 500$ permitió no sólo conocer la historia del lugar a partir de la memoria de los que lo recuerdan, sino imaginarlo como una red de usos públicos que las niñas y niños entendían prioritarios para su zona (Museo Etnográfico, Instituto, Residencia de mayores, Centro Médico, Academia, Supermercado...) (ver Fig.1). 


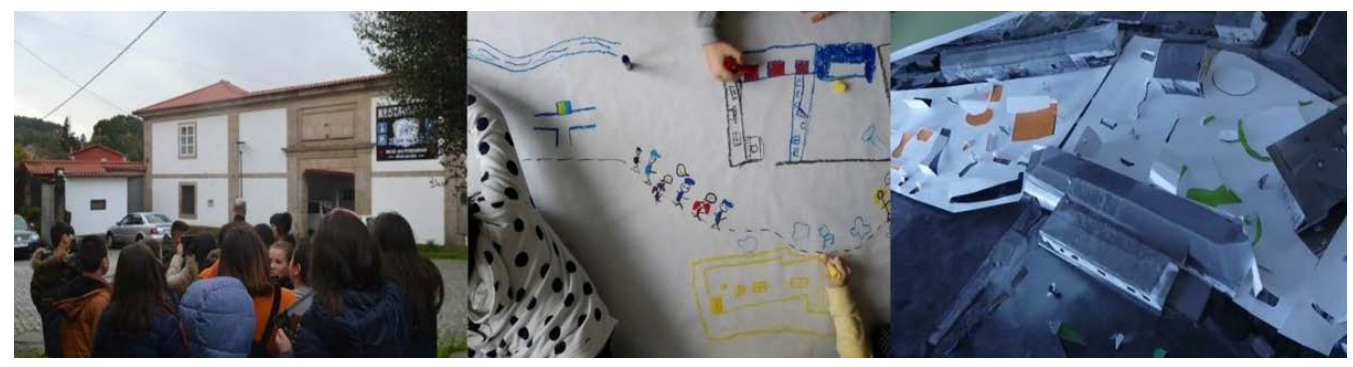

Figura 1. Collage de imágenes de las acciones en los talleres de Lavacolla. Fuente propia.

\subsection{Caso 2. Enfesta. La antigua Estación de Tren. Camino Inglés}

- Centros de realización: Centro Sociocultural de Enfesta y CEIP Casas Novoa.

- Número de participantes: 8 mujeres del grupo de Memoria Activa del Centro Sociocultural/ Una clase de 4 을 de Primaria (16 personas).

- Duración de la propuesta: Sesión de 1 h en el centro sociocultural para las actividades 1 y 2 / 2 sesiones de 50 min para las actividades 3 y 4 desarrolladas en el colegio*.

- Eje principal de desarrollo de la propuesta: Edificio del antiguo ayuntamiento.

* Visita de una de las vecinas al colegio para contar cómo era antes y ahora el núcleo de Enfesta. Realización en aula de una cartografía superpuesta sobre la información recibida.

- Desarrollo de la propuesta: Enfesta sigue teniendo su centro en el edificio del antiguo ayuntamiento, hoy sede del local social donde realizamos las primeras reuniones. El Camino Inglés es la vía principal que lo atraviesa y a ambos lados existían diversos usos y servicios actualmente desaparecidos a partir de los cuales se desarrolló la propuesta en el centro educativo. La cartografía propuesta con los niños y niñas en el centro educativo propone una senda verde a partir de la antigua vía del tren, identifica los antiguos oficios y pone en valor el Castro de Vilasuso y sus muros perimetrales actualmente perdidos entre la vegetación (ver Fig.2).

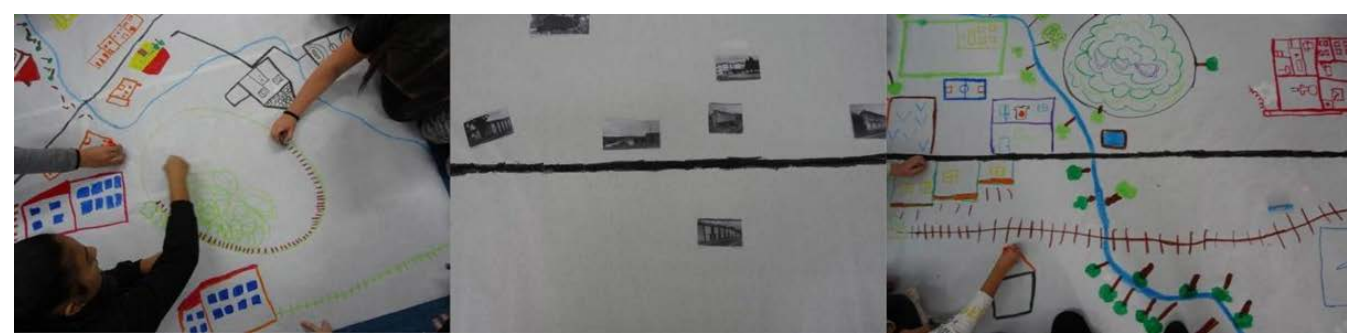

Figura 2. Collage de imágenes realizadas en los talleres de Enfesta. Fuente propia. 


\subsection{Caso 3. Touro. Los oficios del agua. Camino Holandés}

- Centros de realización: Local Social y Casa da Cultura de Touro.

- Número de participantes: 11 mujeres del grupo de Memoria Activa del Local Social/ Grupo de 8 niños y niñas de entre 4 y 8 años que participaban de una actividad de tiempo libre en la Casa de la Cultura.

- Duración de la propuesta: Sesión de 1 h en el local social para las actividades 1 y 2 / 1 sesión de 50 min para las actividades 3 y 4 en la Casa da Cultura*.

- Eje principal de desarrollo de la propuesta: La red fluvial y los oficios.

*Exposición guiada por el técnico del museo etnográfico de Touro sobre fotografías antiguas de los diferentes oficios vinculados al uso, oficios y tratamiento del agua. Realización de una maqueta y cartografía general del ayuntamiento para analizar los cursos fluviales y las actividades que generaban en su entorno inmediato.

- Desarrollo de la propuesta: Touro es denominada la tierra de los mil ríos. Las numerosas arquitecturas del agua que en él se encuentran (molinos, casas de baño, fuentes...) y los oficios vinculados a éstas dan muestra de ello. La propuesta se centró principalmente en el análisis del impacto medioambiental de la mina de Touro sobre la extensa red fluvial del ayuntamiento y en la puesta en valor de los antiguos oficios desaparecidos. Las ferias y fiestas tradicionales eran el epicentro de la actividad social, económica y cultural del ayuntamiento. La cartografía resultante recupera los antiguos caminos de intercambio y los espacios verdes asociados a ellos, con una propuesta dirigida al impulso del Camino Holandés desde una perspectiva sostenible y respetuosa con el medioambiente (ver fig.3).

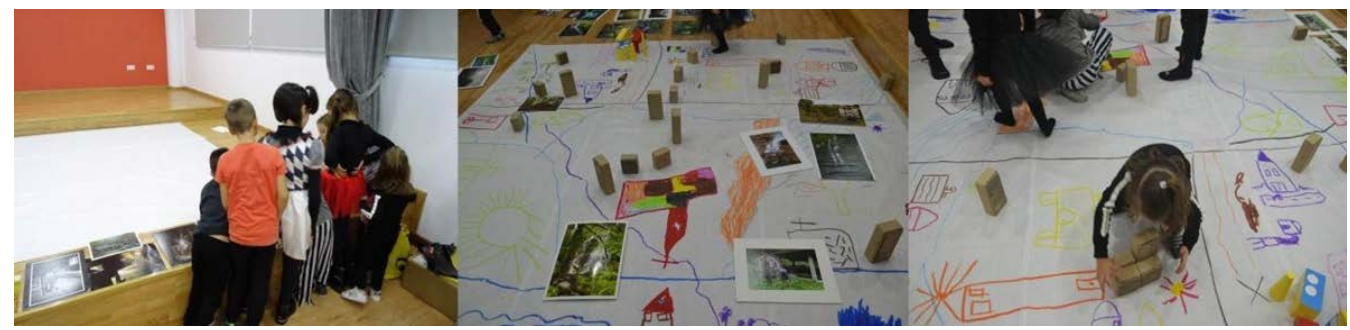

Figura 3. Collage de imágenes realizadas en los talleres de Touro. Fuente propia.

\subsection{Caso 3. Touro. Los oficios del agua. Camino Holandés}

- Centros de realización: Local Social de la Asociación A Mámoa.

- Número de participantes: Grupos de familias intergeneracionales. En total 15 personas de entre 4 y 65 años (9 personas adultas y 6 niñas).

- Duración de la propuesta: Sesión de 1 h en el local de la asociación para las actividades 1 y 2 / 
1 sesión de 1 h 30 min para las actividades 3 y 4*.

- Eje principal de desarrollo de la propuesta: El río Angueira. Petroglifos, caminos y oficios.

* En este caso se realizó una ruta existente en el municipio que discurre paralela al curso del río Angueira y que forma parte de la Ruta de los Petróglifos de Teo. Esta actividad guiada por personas pertenecientes a la asociación permitió no sólo visitar las diferentes estaciones de los petroglifos sino también hablar sobre los antiguos oficios y la riqueza de la biodiversidad de la zona.

- Desarrollo de la propuesta: La red de caminos del ayuntamiento conforma una estructura territorial donde vías romanas, caminos reales y caminos de agra se entrelazan entre pequeñas poblaciones rurales con un patrimonio arqueológico, cultural, natural y social muy extenso. Teo cuenta con numerosas y variadas muestras de petroglifos y mámoas. La propuesta en este caso incorpora la deriva y los cuadernos de viaje como herramientas principales para el análisis del territorio a través de la Ruta por el río Angueira (ver fig.4). En esta ruta descubrimos aldeas, molinos, puentes, caminos históricos e historias de vida de zahoríes, canteros, lavanderas y molineras.
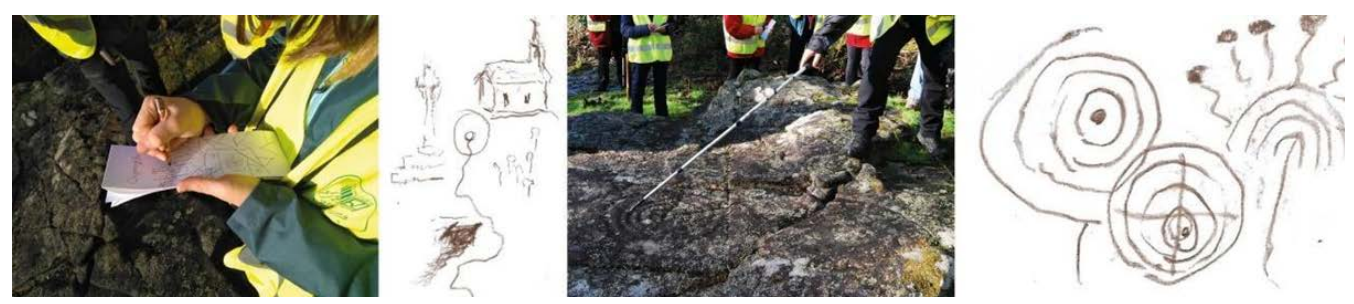

Figura 4. Collage de imágenes realizadas en los talleres de Teo. Fuente propia.

\subsection{Caso 5. Ames. Parcelación y Territorio. Camino Real Fisterra- Muxía}

- Centros de realización: Local Social de Augapesada y CEIP Barouta.

- Número de participantes: 6 mujeres del grupo de Memoria Activa del Local social/ Dos clases de 4을 de Primaria (20 personas).

- Duración de la propuesta: Sesión de 1 h en el local social para las actividades 1 y 2 / 1 sesión de 50 min para las actividades 3 y 4 desarrolladas en el colegio*.

- Eje principal de desarrollo de la propuesta: Los caminos y la concentración parcelaria.

*Análisis de los diferentes caminos que conforman la estructura de las aldeas en las que vivimos y las modificaciones introducidas en el paisaje a través de la aplicación de la concentración parcelaria.

- Desarrollo de la propuesta: Ruta de finalización de la peregrinación Xacobea y trazado histórico de comunicación de la capital compostelana con la costa, el camino real Fisterra-Muxía fue y sigue siendo ruta de intercambio social, económico y cultural. Los cambios territoriales fruto 
de la concentración parcelaria no sólo afectaron al trazado del camino histórico, sino también al trazado de ríos y núcleos de población. La propuesta se centra en este caso en presentar el antes y el después del territorio a través del análisis de fotografías aéreas de la zona. Las niñas y niños conformaron un puzzle cartográfico de sus aldeas de origen, superponiendo los antiguos trazados y riqueza parcelaria a la retícula aplicada por la concentración (ver fig.5).

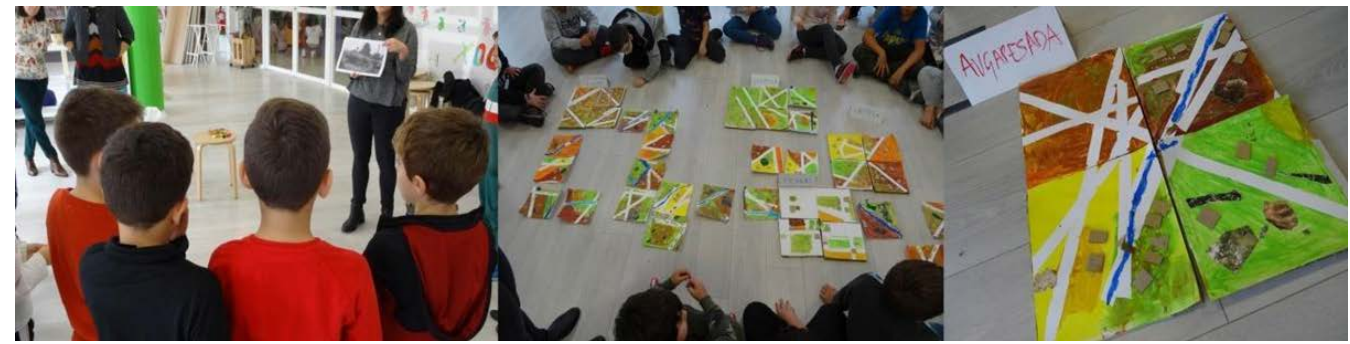

Figura 5. Collage de imágenes realizadas en los talleres de Ames. Fuente propia.

\subsection{Análisis y Discusión. Cartografías de la memoria popular}

Cada actividad realizada representa una subunidad de análisis dentro del conjunto del proyecto. Estas subunidades fueron diseñadas para cada caso concreto según el contexto propio del lugar en el que se llevaron a cabo, manteniendo la estructura y secuencia original presentada. El análisis se centra principalmente en la búsqueda de patrones similares determinantes para el desarrollo y la buena ejecución de las acciones. La participación directa de las personas investigadoras en el desarrollo de la propuesta permite, mediante la observación, las notas extraídas y la revisión de la documentación gráfica que aportan las fotografías tomadas durante la realización de los talleres, la implementación y mejora de las actividades a medida que se va desarrollando el proyecto.

El análisis no sólo se centra en el resultado de dicha implementación, sino que recoge y evalúa los resultados teniendo en cuenta preguntas de investigación expuestas a continuación. Estas preguntas responden a un conjunto de criterios que permiten relacionar la práctica realizada con los objetivos de partida e identificar aquellas experiencias que permitan establecer nuevas formas de relación con el contexto inmediato y presentar una propuesta que pueda ser extrapolada e implementada en futuras ocasiones. Esta evaluación toma como referencia el estudio de análisis realizado por Palacios (2006) sobre el proyecto Art and the built environment. El proyecto partía de una premisa fundamental que era la de analizar y trabajar el entorno local mediante la experiencia directa del lugar y a través de las historias de vida de las personas que lo habitan.

Estas experiencias conectan Educación Ambiental, Educación Patrimonial y otras perspectivas de Educación Basada en el Lugar con la Educación Artística. La Educación Artística se presenta como herramienta fundamental para la investigación, reflexión y cuestionamiento del entorno. Lo que trata de evidenciar la propuesta es la riqueza que aportan este tipo de metodologías artísticas aplicadas de forma interdisciplinar y entendidas como campos abiertos para la expresión en múltiples lenguajes combinables entre sí. 
A continuación presentamos las preguntas de investigación, en relación a los cuatro objetivos específicos presentados en el anterior apartado:

1. ¿Cómo se ha enriquecido el conocimiento del entorno? ¿Se ha hecho su comprensión del entorno más profunda y compleja?

2. ¿Cuáles fueron las fuentes utilizadas para el diseño de la propuesta de actividades? ¿Qué nuevas formas de pensamiento y de conocimiento han sido sugeridas?

3. ¿Cuáles fueron los métodos utilizados en el desarrollo de las propuestas de actividades? ¿Cuáles de ellos han dado mejor resultado y cómo se podrían implementar?

4. ¿Qué tipo de aprendizajes se derivan del estudio directo del entorno rural y qué competencias específicas han adquirido? ¿Cómo podemos fomentar el desarrollo de propuestas similares?

El diseño de la propuesta se utiliza prioritariamente como un método de estudio para poder evaluar el desarrollo e impacto de este tipo de propuestas interdisciplinares que utilizan como herramienta principal de desarrollo actividades vinculadas a la expresión y creación artística. De las grabaciones realizadas durante el desarrollo de la propuesta se obtienen reflexiones y pensamientos que responden a las preguntas anteriores. Los resultados aquí expuestos dan una idea global de las principales cuestiones analizadas. Las narrativas muestran la relación de la interacción grupal entre las diferentes generaciones, proporcionando el enfoque clave para las conclusiones presentadas.

En cuanto a la pregunta de si se ha enriquecido el conocimiento del entorno, los colectivos vecinales y el alumnado crearon un espacio de debate donde se intercambiaron ideas y opiniones sobre los lugares, sus significados y sus experiencias en ellos, recuperando historias de vida y rememorando modos de vivir. Muchos de los relatos sacaron a la luz lugares olvidados por el tiempo, oficios perdidos o cambios en la forma de vida en las últimas décadas; se compartieron emociones y recuerdos personales que confluían con formas de pensar y sentir colectivas, y también se visibilizaron opiniones sobre las necesidades que los propios lugares demandan en la actualidad:

\section{El trabajo de hilar ha desaparecido. También labrar el lino, las devanadoiras, la rueca, el uso, la mazaroca, la estopa y el cerro. Antes se hacía todo en casa: las cestas, los cestos, los cañizos, las gradas, los arados y hasta los ejes de los carros (Fuente: Conversaciones en los centros sociales).}

En cuanto a las fuentes utilizadas, recurrimos a las narraciones de los propios vecinos y las vecinas obtenidas en las reuniones en los centros culturales, en donde cada participante iba aportando datos sobre los lugares. Estos testimonios son claves para trabajar después con el alumnado, ya que aportan información vivencial que no otorgan otro tipo de fuentes escritas. Por otra parte, las rutas guiadas generan un aprendizaje a través de la experiencia en los propios lugares, analizando y observando el entorno físico-natural y social, redescubriendo aspectos del contexto en el que vivimos día a día. 


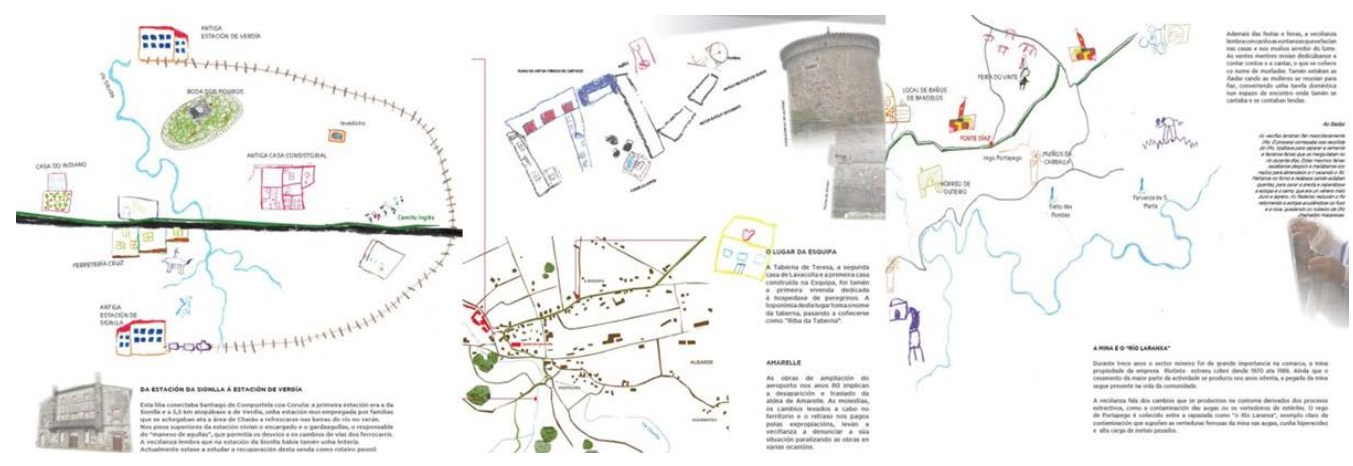

Figura 6. Fotomontaje de los detalles de los paneles realizados. Fuente propia.

Un parque, una fábrica, una fuente, los elementos del entorno cotidiano se convirtieron en los detonantes de la memoria y al mismo tiempo en el vínculo intergeneracional que permitiría relacionar los relatos del pasado con los del presente. En las escuelas se trabajó a partir de las cartografías emocionales de las reuniones con los vecinos y vecinas. Fueron los propios participantes los que contaron al alumnado con sus propias palabras sus vivencias y su relación con los mismos. Los grupos de alumnos trabajaron en los espacios, reconociéndolos y analizándolos in situ.

\section{Aquí se ve muy bien por donde pasaba el camino antiguo y la concentración parcelaria: en la bajada estaba el camino tradicional al molino, que es lo que hay que recuperar (Fuente: Conversaciones durante la ruta por el río Angueira).}

De los métodos utilizados y de las producciones que de ellos se derivan podemos extraer los aprendizajes principales y sus competencias específicas. Trabajar en el propio entorno a través de metodologías basadas en procesos artísticos, como pueden ser las cartografías o los cuadernos de viaje, permitió a cada participante interpretar la realidad poniendo en marcha mecanismos de observación, análisis y reflexión, plasmando los resultados de forma artísticoplástica. A través de las metodologías artísticas pudieron exteriorizar su experiencia, sus emociones y sus opiniones, resignificando sus realidades en relación a los lugares que visitaron. El dibujo, el collage o la fotografía dan cuenta de esta red de interrelaciones patrimoniales. Las cartografías finales sintetizan todas estas narrativas en un relato del pasado y el presente, una suerte de mapas por capas que ofrecen una nueva visión del territorio y constituyen la memoria colectiva.

Experimentar y cuestionar el entorno inmediato nos hace establecer relaciones de significado con los diversos elementos que forman parte del territorio en el que vivimos y del que en muchos casos no somos conscientes. Muchos desconocían el patrimonio tanto material como inmaterial de su entorno, las rutas y visitas a los lugares fueron claves en este proceso.

¿Qué colores hay en nuestro entorno? ¿Son las parcelas de estructura regular o irregular? ¿El río que pasa por mi aldea es el mismo que pasa por la tuya? ¿Cuántos caminos atraviesan mi aldea? ¿Cómo funciona un molino? ¿Cuántos lavaderos conoces? (Fuente: Talleres en los centros educativos). 
Uno de los principales problemas para trabajar la apreciación estética, social y cultural del territorio fue la falta de vocabulario adecuado para describir y comunicar esa experiencia. En muchos casos existía un desconocimiento total de las niñas y niños de términos propios asociados a los oficios, herramientas e incluso elementos físicos y constructivos.

\section{¿Qué aprendisteis? Toda la historia de lo que había antes. ¿Sabíais que existía todo esto? No, pero ahora podemos hacer un mural para explicarle a todo el mundo lo que había y lo que queremos (Fuente: Talleres en los centros educativos).}

Los paneles finales, instalados en cada uno de los centros vecinales en los que se llevó a cabo el proyecto sintetizan la interpretación y análisis del territorio por parte de las personas que lo habitan. Están organizados según los niveles de información y aspectos concretos a tratar en cada lugar de intervención, siendo las producciones de los participantes claves para su comprensión. Estas cartografías finales recogen relatos del pasado y del presente, mediante una superposición de mapas y narrativas que ponen en valor la memoria viva del territorio y por extensión la memoria y los modos de habitar de sus comunidades. Estas Cartografías de la Memoria Popular, reflejan las conexiones entre el pasado y el presente, ponen en valor patrimonios materiales e inmateriales estrechamente vinculados con la forma de relacionarse y de vivir el contexto inmediato recuperando la mirada, las historias de vida y la identidad propia de cada lugar. Los dibujos, fotos y creaciones de los habitantes son necesarios para la comprensión del mapa de la localidad, son estos agentes los encargados de explicar lo que viven día a día en su entorno
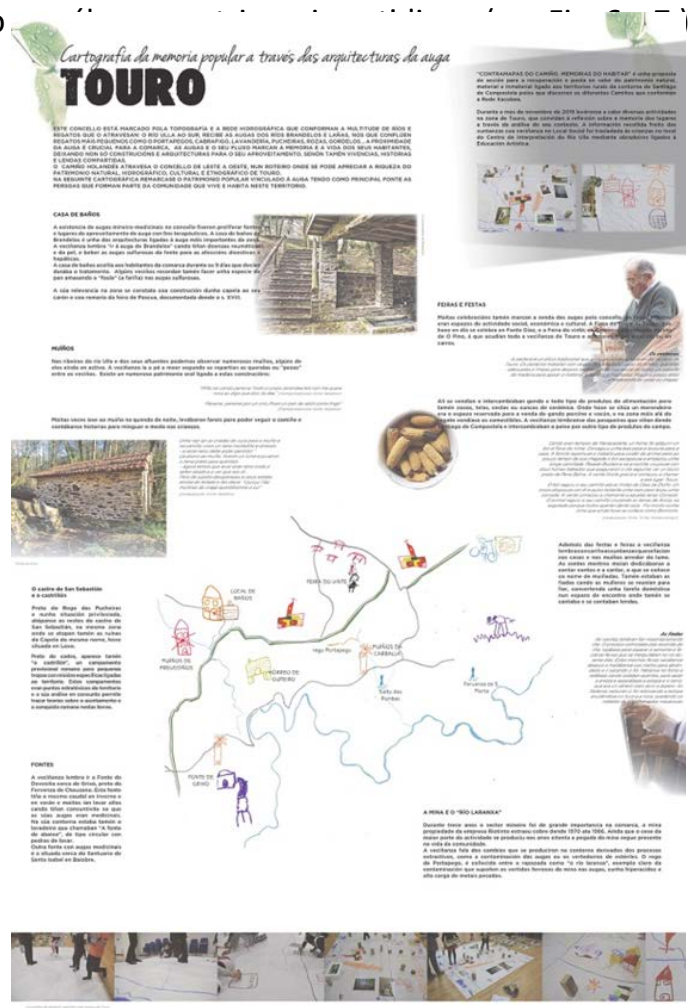

Figura 7. Vista general de uno de los paneles. Fuente propia. 
Esta propuesta de investigación centrada en el análisis de la interpretación del territorio por parte de las comunidades que lo habitan da lugar a un análisis comparativo de las narrativas y producciones obtenidas a través de la maqueta, el collage, el dibujo, la pintura y la fotografía, de las experiencias y percepciones de los participantes que quedan reflejadas en las cartografías. Estas Cartografías de la Memoria Popular forman un relato visual que sintetiza cada una de las experiencias, visibilizando los vínculos relacionales que las personas participantes establecieron con el patrimonio. Finalmente fueron impresas a modo de panel informativo para ser expuestas en el exterior de los centros culturales de las localidades, de forma que tanto los habitantes como los peregrinos fueran los últimos agentes activos en ese proceso de transmisión.

Los criterios para la interpretación de las conclusiones están basados en la documentación recogida en los talleres orales llevados a cabo en los diferentes centros sociales con el grupo de vecinas de edad avanzada y en la medida de análisis de las producciones y narrativas recogidas durante la realización de los talleres en los colegios o centros culturales. EI análisis se centra en mejorar la comprensión de las características distintivas de este tipo de procesos intergeneracionales en los que la información previa recogida en la primera parte de la investigación a través de fuentes informantes locales, es la base para el diseño de las actividades posteriores de desarrollo. En definitiva se trata de evaluar si se produce una transferencia de conocimiento entre generaciones y de si esta transferencia es asimilada por las nuevas generaciones de una forma más efectiva a través de la puesta en práctica de actividades vinculadas a la expresión y creación artística.

El análisis del punto anterior refleja como la experiencia de los participantes da respuesta no sólo a las preguntas de investigación realizadas, sino que también ofrece una visión global muy positiva en relación a los objetivos generales y específicos planteados. Abordar el tema de la construcción colectiva del territorio a través de la secuencia de actividades planteada y su aplicación en diferentes contextos y localizaciones geográficas permite pensar en un método de acción extrapolable a otras situaciones que compartan líneas similares de investigación. El primer contacto con los informantes locales es fundamental para el desarrollo y permite la participación consciente de la persona investigadora que trasciende su papel de observador y se convierte en un agente activo parte de la investigación. Este papel es clave en el desarrollo de metodologías educativas vinculadas a la investigación en educación artística, donde el proceso abierto y flexible de la propia investigación permite ajustarlo en diseño y dimensiones a cada unidad específica de análisis. Este proceso rizomático de construcción del conocimiento garantiza a su vez la transferibilidad de la propuesta y su credibilidad, siendo las conclusiones de un estudio de estas características generalizables más allá de los casos concretos analizados.

Partir de la experiencia vivencial y del contexto local como herramientas principales de diseño posibilita la aplicación del esquema de actividades propuesto en otros contextos con resultados similares. Son necesarios programas complementarios que trabajen desde el entendimiento fundamental del aprendizaje a través de la experiencia y del conocimiento compartido. La educación basada en el lugar y la aplicación de la metodología artística aplicada son recursos que permiten la aproximación al conocimiento del entorno desde un aprendizaje activo y colaborativo. En este sentido, las diferentes propuestas son presentadas como material destinado a facilitar nuevas formas de mirar y experimentar el contexto, centrándose fundamentalmente en las relaciones intergeneracionales que posibilitan la transmisión real del aprendizaje basado 
en la experiencia del lugar y en su identidad. Se trata en definitiva de impulsar la acción creativa y comunitaria como productora de cultura a través de las diferentes lecturas, reflexiones y análisis sobre el patrimonio que nos rodea.

El artículo "Contramapas del Camino. Memorias del Habitar" se desarrolla en referencia a la investigación realizada dentro del proyecto del mismo nombre perteneciente al programa $\mathrm{O}$ teu Xacobeo, impulsado por la Xunta de Galicia. Este proyecto fue presentado desde la USC (Facultade de Ciencias de la Educación de Santiago de Compostela), por el grupo de investigación Liter 21, grupo al cual pertenecemos las tres autoras y del que Carmen Franco Vázquez es la coordinadora e IP.

El diseño y la puesta en práctica de la propuesta, así como el registro de las experiencias y su análisis posterior dentro de la estructura del artículo fue competencia de Estella Freire Pérez y Candela Rajal Alonso. Ambas somos alumnas del programa de doctorado en Educación y Carmen Franco es directora común, por lo que el trabajo último de revisión de la propuesta, estructura y montaje del artículo también le corresponde a ella.

En resumen los porcentajes serían:

Estella L. Freire Pérez. Autora de correspondencia. 40\%

Candela Rajal Alonso. Coautora. 40\%

Carmen Franco Vázquez. Coautora. 20\%

\section{REFERENCIAS}

Adams, E. y Ward, C. (1982). Art and Built Environment: a teacher's approach. London, UK: Longman.

Adams, E. (1989). Learning to see. Journal of Art \& Design Education, 8(2), 183-186.

Adams, E. (2008). Art and design education and the built environment. Art, Community and Environment: Educational Perspectives, 7, 125.

Adams, E. (2012). Education, environment, art: A new year: new opportunities. En Commissioned and published by ixia, the public art think tank. Recuperado de http:// www.publicartonline.org.uk/whatsnew/news/article. php/EDUCATION,+ENVIRONMENT,+ART+-+A+new+year+new+opportunities.htm

Aldrey Vázquez, J.A. y del Río Franqueira, D. (2014). Crónica 
de un envellecemento anunciado: Galicia 2015-2030. Cambio demográfico e socio territorial en un contexto de crisis. XIV Congreso Nacional de Poboación, AGE (pp.69-82). Sevilla: Grupo de Poboación da Asociación de Geógrafos Españoles.

Arévalo, J. M. (2009). El patrimonio como memoria social y representación colectiva. La intangibilidad de los bienes culturales. En Medina García, E., Arévalo, J.M., Gómez-Ullate García de León, M. y Lagunas Arias, D. (coords.), Fronteras, patrimonio y etnicidad en Iberoamérica. (pp. 171-195). Madrid, España: Signatura Demos.

Assmann, J. (2010). Communicative and Cultural Memory. A Companion to Cultural Memory Studies, 109-118.

Blanco, V. y Cidrás, S. (2019). Educar a través da arte. Cara a unha escola imaxinada. Pontevedra, España: Kalandraka.

Barone, T. (2006). Arts-based educational research then, now, and later. Studies in Art Education, 48(1), 4-8.

Barone. T. y Eisner E. (2006) Arts-Based Educational Research. En J. Green, C. Grego y P. Belmore (Eds.), Handbook of Complementary Methods in Educacional Research. (pp.95-109). Mahwah, New Jersey, EEUU: AERA.

Denzin, N. y Lincoln, Y. (1994). Handbook of qualitative research. London, UK: Sage.

Dewey, J. (2004 [1938]). Experiencia y Educación. Madrid, España: Biblioteca Nueva.

Dewey, J. (2008 [1934]). El arte como experiencia. Barcelona, España: Paidós.

Fontal, O. (2003). La educación patrimonial. Gijón, España: Trea.

Fontal, O., García-Ceballos, S., Aso Morán, B. y Martínez, M. (2017). Patrimonios, objetos e historias de vida. Análisis de propuestas educativas desde el Observatorio de Educación Patrimonial en España. MIDAS [Online], 8. Recuperado de http:// journals.openedition.org/midas/1310

Fontal, O. y Marín-Cepeda, S. (2018). Nudos patrimoniales. Análisis de los vínculos de las personas con el patrimonio personal. Arte, individuo y sociedad, 30(3), 483-500.

Gold, J. y Goodey, B. (1983). Behavioural and perceptual 
geography, Progress in human geography, 7(4), 578-586.

Gruenewald, D. y Smith, G. (Eds.) (2008). Creating a movement to ground learning in place. En: Place-based education in the global age: Local diversity (pp. 345-358). London, UK: Routledge.

Halbwachs, M. (2004). La memoria colectiva. Zaragoza, España: Prensas Universitarias de Zaragoza.

Illeris, H. (2012). Nordic contemporary art education and the environment: Constructing an epistemological platform for Art Education for Sustainable Development (AESD), InFormation, 1(2), 77-93.

Instituto Galego de Estadística (2020). Proxeccións de poboación a curso prazo. 2018-2033. Variables demográficas proxectadas. Recuperado de https://www.ige.eu/igebdt/esqv. jsp?ruta=verTabla.jsp?OP $=1 \& B=1 \& M=\& C O D=9285 \& R=9928[12]$; $2[11] ; 0[2018] \& C=1[0] \& F=\& S=\& S C F=$

Instituto Nacional de Estadística (2012). Cifras INE. Boletín Informativo do Instituto Nacional de Estadística. Envellecemento Activo. Recuperado de https://www.ine.é/ss/Satellite?L=\&c=INE CifrasINE_C\&cid=1259940189717\&p=1254735116567\&pagenam e=ProductosYServicios\%2FPYSLayout

Jokela, T. S., y Coutts, G. (2018). The North and the Arctic: A laboratory af art and design education for sustainability. In T. Jokela, \& G. Coutts (Eds.), Relate North: Art \& design for education and sustainability (pp. 98-117). Lapland University Press.

Mantere, M. H. (1992). Ecology, environmental education and art teaching, En: L. Piironen (Ed.) Power of images (pp. 17-26). Helsinki, Finland: INSEA Research Congress, Association of Art Teachers.

Marín, R., (2011). Las investigaciones en educación artística y las metodologías artísticas de investigación en educación: temas, tendencias y miradas, Educação, 34(3), 271-285.

McNiff, S. (2008). Arts-based research. En J. Green y A.L. Cole (Eds.), Handbook of Complementary Methods in Educacional Research. Handbook of arts in qualitative research (pp. 29-40). California, EEUU: Sage Publications In.

Mullen, C., (2003). A self fashioned gallery of aesthetic practice. Qualitative Inquiry, 9(2), 165-182. 
Muñoz Viñas, S. (2010). Teoría contemporánea de la Restauración. Madrid, España: Síntesis.

Neperud, R. (1978). The what and why of environmental design education. Studies in Art Education, 31(4), 4-7.

Palacios, A. (2005). La comprensión del entorno construido desde la educación artística. Una propuesta para educación primaria y formación inicial del profesorado. Madrid, España: Universidad Complutense de Madrid.

Palacios, A. (2006). Educación artística y ambiental: un estudio del caso británico a través del proyecto Art and the Built Environment. Arte, Individuo y Sociedad, 18, 57-76.

Samaja, J. (1994) Epistemología y Metodología. Elementos para una teoría de la investigación científica. Buenos Aires, Argentina: Eudeba.

Sánchez Pardo, J. C. (2013). Bases para el análisis geohistórico del poblamiento rural tradicional en Galicia. BAGE. Boletín de la Asociación de Geógrafos Españoles, 62, 75-99.

Sullivan, G. (2004). Art Practice as Research Inquiry in the Visual Arts. New York, EEUU: Teachers College, Columbia University.

Taylor, S. y Bodgan, R. (1996). Introducción a los métodos cualitativos de investigación. Barcelona, España: Paidós.

Unesco (2003). Convención para la Salvaguardia del Patrimonio Cultural Inmaterial. Recuperado de https://ich. unesco.org/es/convenci\%C3\%B3n

Muñoz-Viñas, S. (2010). Teoría contemporánea de la restauración. Madrid, España: Editorial Síntesis.

Yin, R. (2003). Case study research: Design and methods. London, UK: Sage. 